www.nature.com/hr

\title{
Physical activity modifies the association between CYBA gene polymorphisms and small artery elasticity in a Chinese population
}

\author{
Zhenyan Zhu ${ }^{1,5}$, Haifeng Zhang ${ }^{1,5}$, Wenming Yao ${ }^{1}$, Ningxia Liang ${ }^{1}$, Lei Gong ${ }^{2}$, Zhijian Yin ${ }^{3}$, Fukuan Chen ${ }^{4}$, \\ Kejiang $\mathrm{Cao}^{1}$ and $\mathrm{Xinli} \mathrm{Li}^{1}$
}

Emerging evidence suggests that increased superoxide production is responsible for a significant proportion of endothelial dysfunction. The relationship between variants of the CYBA gene and cardiovascular diseases is currently debated. In the present study, we investigated the influence of CYBA polymorphisms (rs1049255 and rs7195830) on arterial elasticity in a Chinese population. In the $\mathbf{2 1 7 8}$ participants enrolled in the GaoYou study, we measured large artery elasticity (C1) and small artery elasticity (C2) non-invasively, genotyped the CYBA polymorphisms and calculated energy expenditure. The AA genotype of the rs 1049255 polymorphism was associated with a lower $C 2$ than were the GG/AG genotypes $(5.31 \pm 0.11$ vs. $5.52 \pm$ $\left.0.06 \mathrm{~m} \mathrm{~mm} \mathrm{Hg}{ }^{-1} \times 100 ; P=0.01\right)$. Further analyses revealed an interaction between CYBA polymorphisms and physical activity with respect to $C 2(P=0.007$ for rs 1049255 and $P=0.038$ for rs7195830). In less physically active participants, the $A A$ genotype of the rs 1049255 polymorphism was associated with a significantly lower $C 2$ than the GG/AG genotypes $(4.69 \pm 0.16$ vs. $5.26 \pm 0.19 \mathrm{ml} \mathrm{mm} \mathrm{Hg}{ }^{-1} \times 100 ; P=0.008$ ). In physically active participants, the GG/AG genotypes of $\mathrm{rs} 7195830$ polymorphism were correlated with higher $C 2$ values than the AA genotype $\left(5.84 \pm 0.08\right.$ vs. $5.08 \pm 0.32 \mathrm{ml} \mathrm{mm} \mathrm{Hg}^{-1} \times 100$; $P=0.049)$. Haplotype analyses revealed higher $C 2$ values in rs1049255G-rs7195830G carriers $(P=0.0015)$. In conclusion, the rs1049255 and rs7195830 polymorphisms of the CYBA gene were associated with C2 in a Chinese population; physical activity could modify this genetic effect.

Hypertension Research (2012) 35, 739-744; doi:10.1038/hr.2012.23; published online 23 February 2012

Keywords: arterial Celasticity; CYBA gene; endothelial function; general population; physical activity

\section{INTRODUCTION}

Endothelial dysfunction is considered to be the earliest event in a diverse range of cardiovascular diseases. ${ }^{1}$ Recent studies have indicated that the large artery elasticity index $(\mathrm{C} 1)$ and the small artery elasticity index (C2) are related to endothelial function; ${ }^{2,3}$ lower values of $\mathrm{C} 1$ and $\mathrm{C} 2$ indicate worse arterial elasticity. Remarkably, C2 has been approved to be a predictive value for cardiovascular disease events. ${ }^{4}$ Recent studies have shown that arterial elasticity can be affected by regular physical activity ${ }^{5}$ and is associated with heredity. ${ }^{6}$

Oxidative stress is thought to have an important role in endothelial dysfunction. ${ }^{1}$ Reactive oxygen species are produced by a wide variety of enzymatic sources ${ }^{7}$ among which nicotinamide adenine dinucleotide phosphate oxidase appears to be a predominant contributor of superoxide anion. The P22phox subunit, encoded by the CYBA gene located on chromosome 16q24, is a crucial component of the nicotinamide adenine dinucleotide phosphate oxidase system and has a key role in superoxide anion production. ${ }^{8}$ Several variants of the CYBA gene have been identified. Among these, rs1049255 (A640G) has been widely discussed in the context of cardiovascular diseases; however, the results have been conflicting. ${ }^{9-12}$ For rs7195830, there have been few studies related to cardiovascular diseases. Rather, rs7195830 has been mentioned in studies on non-Hodgkin lymphoma and cervical cancer as an oxidative stress-related locus. ${ }^{13,14}$

Therefore, in the present study, we hypothesized that the rs1049255 and rs7195830 polymorphisms of the CYBA gene are associated with arterial elasticity indices in a large cohort of apparently healthy Chinese individuals across a wide age range. As physical activity may influence oxidative stress biomarkers, ${ }^{15}$ we further explored whether physical activity could modify the genetic effect.

\section{METHODS}

Study population

Stratified random sampling was used to select a representative sample in the towns of Baqiao and Songqiao in Gaoyou, a city 170-km north of Nanjing,

${ }^{1}$ Department of Cardiology, The First Affiliated Hospital, Nanjing Medical University, Nanjing, China; ${ }^{2}$ The Health Bureau of Gaoyou, Gaoyou, China; ${ }^{3}$ The Centre Clinics of Baqiao, Gaoyou, China and ${ }^{4}$ The Centre Clinics of Songqiao, Gaoyou, China

${ }^{5}$ These authors contributed equally to this work.

Correspondence: Professor X Li, Department of Cardiology, The First Affiliated Hospital, Nanjing Medical University, Guangzhou Road 300, Nanjing 210029, China. E-mail: xinli3267@yeah.net

Received 1 October 2011; revised 12 December 2011; accepted 11 January 2012; published online 23 February 2012 
China. The sample was stratified by sex and age on the basis of population data obtained from the local authority. Finally, 5000 subjects in Baqiao and 1000 subjects in Songqiao aged 18 to 75 years old were selected and invited to participate in the study. In the period from January to May 2010, 4536 subjects participated in the present study, including 3918 subjects in Baqiao and 618 subjects in Songqiao; the response rates were $78.4 \%$ and $61.8 \%$, respectively.

In order to obtain an apparently healthy population, individuals with hypertension (systolic blood pressure $\geqslant 140 \mathrm{~mm} \mathrm{Hg}$ or diastolic blood pressure $\geqslant 90 \mathrm{~mm} \mathrm{Hg}$ or on antihypertensive medications; $n=2168$ ), diabetes (based on a clinical history or serum glucose $\geqslant 7.0 \mathrm{mmoll}^{-1}$ or the use of antidiabetic medications; $n=105$ ) and other cardiovascular diseases (based on a clinical history or evidence on examination or use of medication; $n=45$ ) were excluded from the present study. Individuals were also excluded owing to missing information on arterial elasticity indices $(n=37)$ or genotypes of both polymorphisms $(n=3)$. Thus, 2178 participants were statistically analyzed. The Institutional Review Board of the First Affiliated Hospital, Nanjing Medical University, approved the study protocol. Written informed consent was obtained from each participant before data collection.

\section{Measurement of phenotypes}

Experienced observers administered a standardized questionnaire to collect information on medical history, smoking habits, alcohol consumption and use of medications. Each participant's blood pressure was measured three times consecutively by conventional sphygmomanometry after the participants had rested for at least $5 \mathrm{~min}$ in the sitting position. These three readings were averaged for analysis. The mean arterial pressure was calculated by this equation: $((2 \times$ diastolic blood pressure $)+$ systolic blood pressure $) / 3$. The body weight, body height, and waist and hip circumferences were measured. The body mass index was calculated as the ratio of the body weight in kilograms to the square of the height in meters, and the waist-to-hip ratio was the smallest circumference at the waist divided by the largest circumference at the hip.

\section{Measurement of energy expenditure}

Habitual physical activity was assessed by a validated ${ }^{16,17}$ self-report questionnaire called the International Physical Activity Questionnaire, which mainly focused on two questions. The first question asked about usual physical activity at work was classified into four categories: sedentary, standing, manual work and heavy manual work. The second question asked about the amount of time spent (in hours per week) separately in two activities: walking and other recreational physical activity (for example, keep fit, jogging and swimming). The average time spent daily in walking and recreational activity was estimated as the simple mean of the total hours spent per week, divided by seven. We calculated the energy expenditure engaging in physical activity based on each participant's body weight, the time devoted to work and sports, and the metabolic equivalent according to the degree of the occupational activity and the type of recreational physical activity. ${ }^{18-20}$ The median of energy expenditure in our population was $2528 \mathrm{kcal}$ per day; thus, as reported previously, ${ }^{20}$ subjects with energy expenditure less than the median were identified as less physically active, whereas subjects with energy expenditure above the median were identified as physically active.

\section{Measurement of serum biomarkers}

Venous blood was sampled after overnight fasting. We measured the levels of serum glucose, serum triglycerides, serum total cholesterol and serum highdensity lipoprotein (HDL) cholesterol using an automated enzymatic method (Chemistry Analyzer AU2700, Olympus Medical Engineering Company, Tokyo, Japan). The serum low-density lipoprotein (LDL) cholesterol concentration was calculated using the Friedewald formula.

\section{Measurement of arterial elasticity indices}

We used the HDI/PulseWave CR-2000 Research CardioVascular Profiling System (Hypertension Diagnostics, Inc., Eagan, MN, USA) to measure the elasticity of the arterial system non-invasively by pulse wave analysis using the Windkessel model. ${ }^{21}$ This model generates elasticity indices of the large arteries $\left(\mathrm{C} 1\right.$, capacitive arterial compliance, units in $\left.\mathrm{ml} \mathrm{mm} \mathrm{Hg}^{-1} \times 10\right)$ and of the small arteries $(\mathrm{C} 2$, reflective or oscillatory arterial compliance, units in $\mathrm{ml} \mathrm{mm} \mathrm{Hg}^{-1} \times 100$ ) by reproducing arterial pressure waveforms including both exponential and oscillatory pressure decay observed during the diastolic decay portion of the cardiac cycle. This technique has been previously described in detail, ${ }^{22}$ and was recently used in clinical research in several countries including China. ${ }^{23,24}$ The reproducibility of this technique has also been approved in a recent study. ${ }^{25}$ Each participant's blood pressure was measured three times consecutively after resting for at least $5 \mathrm{~min}$ in the supine position and the three readings were averaged for analysis. One observer (ZZ) performed all of the arterial measurements.

\section{Genotyping}

In this study, we chose two single-nucleotide polymorphisms (SNPs) (rs1049255 and rs7195830 in the $3^{\prime}$-untranslated region) that, according to the literature, may be involved in endothelial dysfunction or may be associated with oxidative stress, and are in the same haplotype block according to the Chinese database in HAPMAP (http://hapmap.ncbi.nlm.nih.gov).

Whole-venous blood was drawn into ethylenediamine tetraacetic acid tubes and then stored at $-80^{\circ} \mathrm{C}$. Genomic DNA was extracted from white blood cells using a commercially available kit and the BioRobot M48 Workstation (Qiagen Inc., Hilden, Germany) according to the manufacturer's instructions. PCR for the rs1049255 and rs7195830 polymorphisms was performed in 384-well plates following a standard protocol $^{26}$ for TaqMan MGB probes (rs1049255: C_7516913_10, ABI, Foster City, CA, USA; rs7195830: C_28959511_10, $\mathrm{ABI})$. After the PCR was finished, endpoint fluorescence was measured, and allelic discrimination was performed using a PRISM 7900HT Sequence Detection System (ABI) and a LightCycler 480 Real-Time PCR System (Roche, Rotkreuz, Switzerland). Genotyping quality control was performed in $10 \%$ of the samples by duplicate checking (rate of concordance in duplicates $>99 \%$ ).

\section{Statistical analysis}

For database management and statistical analysis, we used SAS software, version 9.1.3 (SAS Institute, Cary, North Carolina, USA). We reported the central tendency and spread of normally and non-normally distributed data as the mean \pm s.d. and as the median with the interquartile range, respectively. Hardy-Weinberg equilibrium was assessed using the $\chi^{2}$ test. We compared the means and proportions using the $t$-test, one-way analysis of variance and $\chi^{2}$ test, respectively. We performed multiple regression analysis to select significant correlates of arterial elasticity indices ( $\mathrm{C} 1$ and $\mathrm{C} 2$ ). In the subsequent multiple regression analysis that compared the arterial elasticity indices across genotypes for the two CYBA gene polymorphisms, we included all significant correlates as covariates and the recessive models (major homozygous along with heterozygous genotype as a reference) were typically used. Haplotypes were constructed with Haploview and SNPHAP program (http://wwwgene.cimr.cam.ac.uk/clayton/software). Haplotypes were only assigned when the probability was greater than $95 \%$ based on the SNPHAP program, and haplotypes with a frequency of $<1 \%$ were dropped from the analyses. For each haplotype, we compared the arterial elasticity indices between carriers and noncarriers, using the latter as a reference. As for the analysis of the single SNPs, multiple regression analyses were also used in haplotype analyses adjusted for the same covariates. $P$-value of $<0.05$ was considered statistically significant.

\section{RESULTS}

\section{Characteristics of the study population}

The 2178 subjects included 857 men (39.3\%) and 1321 women (60.7\%); the age ranged from 18 to 75 years. The characteristics of all subjects stratified by both polymorphisms are shown in Table 1. The waist-to-hip ratio was higher in subjects with the AA genotype in the rs1049255 polymorphism, but none of the variables differed between genotypes of the rs7195830 polymorphism.

\section{Genotype frequencies and haplotype reconstruction}

The genotype frequencies of both loci are also shown in Table 1. The A allele frequency was $41.9 \%$ for rs 1049255 polymorphism and was $22.2 \%$ for rs7195830 polymorphism. The frequencies of the rs1049255 and rs7195830 genotypes did not deviate from Hardy-Weinberg equilibrium ( $P=0.07$ and 0.79 , respectively) or differ with respect to the subgroups divided by energy expenditure. The two SNPs were in 
Table 1 Characteristics of all subjects stratified by rs1049255 and rs7195830 genotype

\begin{tabular}{|c|c|c|c|c|c|c|}
\hline \multirow[b]{2}{*}{ Characteristics } & \multicolumn{3}{|c|}{ rs1049255 } & \multicolumn{3}{|c|}{ rs7195830 } \\
\hline & $G G(n=747)$ & $A G(\mathrm{n}=1007)$ & $A A(\mathrm{n}=398)$ & $G G(n=1315)$ & $A G(\mathrm{n}=745)$ & $A A(\mathrm{n}=109)$ \\
\hline Age, years & $47.2 \pm 12.1$ & $47.5 \pm 12.2$ & $47.5 \pm 12.1$ & $47.4 \pm 12.1$ & $47.4 \pm 12.4$ & $48.4 \pm 12.1$ \\
\hline Body mass index, $\mathrm{kg} \mathrm{m}^{-2}$ & $23.6 \pm 2.9$ & $23.7 \pm 2.9$ & $24.0 \pm 3.0$ & $23.7 \pm 2.9$ & $23.8 \pm 3.0$ & $23.4 \pm 2.8$ \\
\hline Waist-to-hip ratio & $0.84 \pm 0.06$ & $0.83 \pm 0.06$ & $0.85 \pm 0.07^{*}$ & $0.84 \pm 0.06$ & $0.84 \pm 0.06$ & $0.84 \pm 0.06$ \\
\hline \multicolumn{7}{|l|}{ Arterial measurements } \\
\hline Systolic blood pressure, $\mathrm{mmHg}$ & $121.5 \pm 78.5$ & $122.0 \pm 10.0$ & $121.5 \pm 10.0$ & $121.7 \pm 9.8$ & $121.8 \pm 10.2$ & $122.0 \pm 10.0$ \\
\hline Diastolic blood pressure, $\mathrm{mmHg}$ & $78.5 \pm 6.6$ & $78.6 \pm 6.5$ & $77.9 \pm 6.9$ & $78.3 \pm 6.6$ & $78.7 \pm 6.6$ & $78.3 \pm 6.9$ \\
\hline Mean arterial pressure, $\mathrm{mmHg}$ & $92.8 \pm 6.8$ & $93.0 \pm 6.9$ & $92.4 \pm 7.2$ & $92.8 \pm 6.9$ & $93.0 \pm 7.1$ & $92.9 \pm 7.0$ \\
\hline \multicolumn{7}{|l|}{ Blood biochemistry } \\
\hline LDL cholesterol, mmol I-1 & $2.78 \pm 0.65$ & $2.80 \pm 0.66$ & $2.84 \pm 0.70$ & $2.80 \pm 0.66$ & $2.78 \pm 0.64$ & $2.93 \pm 0.74$ \\
\hline Triglycerides, mmoll-1a & $1.04(0.77-1.48)$ & $1.07(0.79-1.53)$ & $1.10(0.81-1.61)$ & $1.07(0.79-1.54)$ & $1.05(0.78-1.49)$ & $1.03(0.75-1.52)$ \\
\hline Male sex, $n(\%)$ & $295(39.5)$ & $379(37.6)$ & $171(42.9)$ & 525 (39.9) & $286(38.4)$ & $42(38.5)$ \\
\hline Current smoking, $n(\%)$ & $206(27.6)$ & $263(26.1)$ & $108(27.1)$ & $347(26.4)$ & $209(28.1)$ & $29(26.6)$ \\
\hline Alcohol intake, $n(\%)$ & $115(15.4)$ & $151(15.0)$ & $64(16.1)$ & $197(15.0)$ & $119(16.0)$ & $17(15.6)$ \\
\hline
\end{tabular}

Abbreviations: HDL, high-density lipoprotein; LDL, low-density lipoprotein.

aThe values are the median (interquartile range). ${ }^{*} P=0.001$.

linkage disequilibrium $\left(\mathrm{D}^{\prime}=0.916, r^{2}=0.175\right)$; the estimated population frequencies for the haplotypes are shown in Table 3.

\section{Multiple regression analysis of arterial elasticity indices}

With multiple regression analysis, both $\mathrm{C} 1$ and $\mathrm{C} 2$ were higher in men than in women and were inversely correlated with age, smoking status, body mass index, waist-to-hip ratio, heart rate, systolic and diastolic blood pressure, concentration of LDL cholesterol, total cholesterol and triglycerides, and were positively correlated with the concentration of HDL cholesterol (Table 2).

\section{Association between arterial elasticity indices and CYBA polymorphisms}

For rs1049255, the AA genotype was associated with significantly lower values of $\mathrm{C} 2$ compared with the GG and AG genotypes

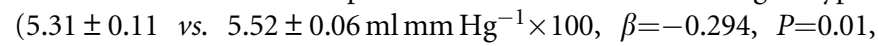
Figure 1a) in the study population, but there was no significant association between genotypes and $\mathrm{C} 1$ (13.76 \pm 0.22 vs. $13.85 \pm$ $\left.0.11 \mathrm{ml} \mathrm{mm} \mathrm{Hg}{ }^{-1} \times 10, \beta=-0.263, P=0.24\right)$. For rs7195830, neither C1 $\quad\left(13.63 \pm 0.47 \quad\right.$ vs. $13.83 \pm 0.10 \mathrm{ml} \mathrm{mm} \mathrm{Hg}^{-1} \times 10, \quad \beta=-0.210$, $P=0.60)$ nor $\mathrm{C} 2 \quad\left(5.09 \pm 0.25\right.$ vs. $5.49 \pm 0.05 \mathrm{ml} \mathrm{mm} \mathrm{Hg}^{-1} \times 100$, $\beta=-0.323, P=0.11$, Figure $1 \mathrm{~b}$ ) was related to the genotype in this population.

\section{Associations between CYBA haplotypes and arterial elasticity indices}

The haplotypes constructed on the basis of the two SNPs showed that the rs1049255G-rs7195830G haplotype carriers were associated with higher C2 values, while the other two haplotypes (rs1049255Ars7195830G and rs1049255G-rs7195830A) did not display such an association. In line with the single SNP analyses, neither of the haplotypes was associated with C1 (Table 3).
Interaction between CYBA polymorphisms and physical activity In further analyses, we found that both polymorphisms had a significant interaction with physical activity in relation to $\mathrm{C} 2$ ( $P=0.007$ for rs1049255, $P=0.038$ for rs7195830) after adjustment for age, sex, heart rate, smoking status, waist-to-hip ratio, body mass index, systolic blood pressure, diastolic blood pressure, total cholesterol level, HDL cholesterol level, LDL cholesterol level and triglyceride levels, while no such interaction was found in relation to $\mathrm{C} 1(P=0.23$ for rs1049255, $P=0.24$ for rs7195830).

In less physically active participants, the AA genotype of the rs1049255 polymorphism was significantly associated with a lower $\mathrm{C} 2 \quad\left(4.69 \pm 0.16 \quad\right.$ vs. $\quad 5.26 \pm 0.19 \mathrm{ml} \mathrm{mm} \mathrm{Hg}{ }^{-1} \times 100, \quad \beta=-0.421$, $P=0.008$, Figure 1a) than the GG and AG genotypes, but this relationship in the physically active participants was not significant

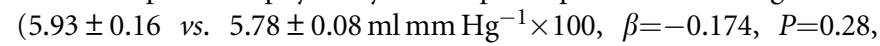
Figure 1a). For rs7195830, the GG and $A G$ genotypes had a higher $\mathrm{C} 2$ compared with the AA genotype (5.84 \pm 0.08 vs. $5.08 \pm 0.32 \mathrm{ml} \mathrm{mm} \mathrm{Hg}^{-1} \times 100, \beta=-0.528, P=0.049$, Figure $\left.1 \mathrm{~b}\right)$ in physically active participants, but there was no such association in less physically active participants $(5.15 \pm 0.08$ vs. $5.11 \pm$ $0.38 \mathrm{ml} \mathrm{mm} \mathrm{Hg}^{-1} \times 100, \beta=-0.138, P=0.65$, Figure $\left.1 \mathrm{~b}\right)$. Genetic associations with $\mathrm{C} 1$ were not significant in either of the subgroups $(P>0.10)$.

\section{DISCUSSION}

To our knowledge, this is the first general-population-based study of apparently healthy subjects to investigate the relationship between variants of the CYBA gene and vascular properties using the $C 1$ and $\mathrm{C} 2$ indices. Our main findings were that the AA genotype of the rs1049255 polymorphism was independently associated with C2 and that physical activity may modify this association. For haplotype analyses, carriers of haplotype rs1049255G-rs7195830G were found 
Table 2 Multiple regression analysis of arterial elasticity indices

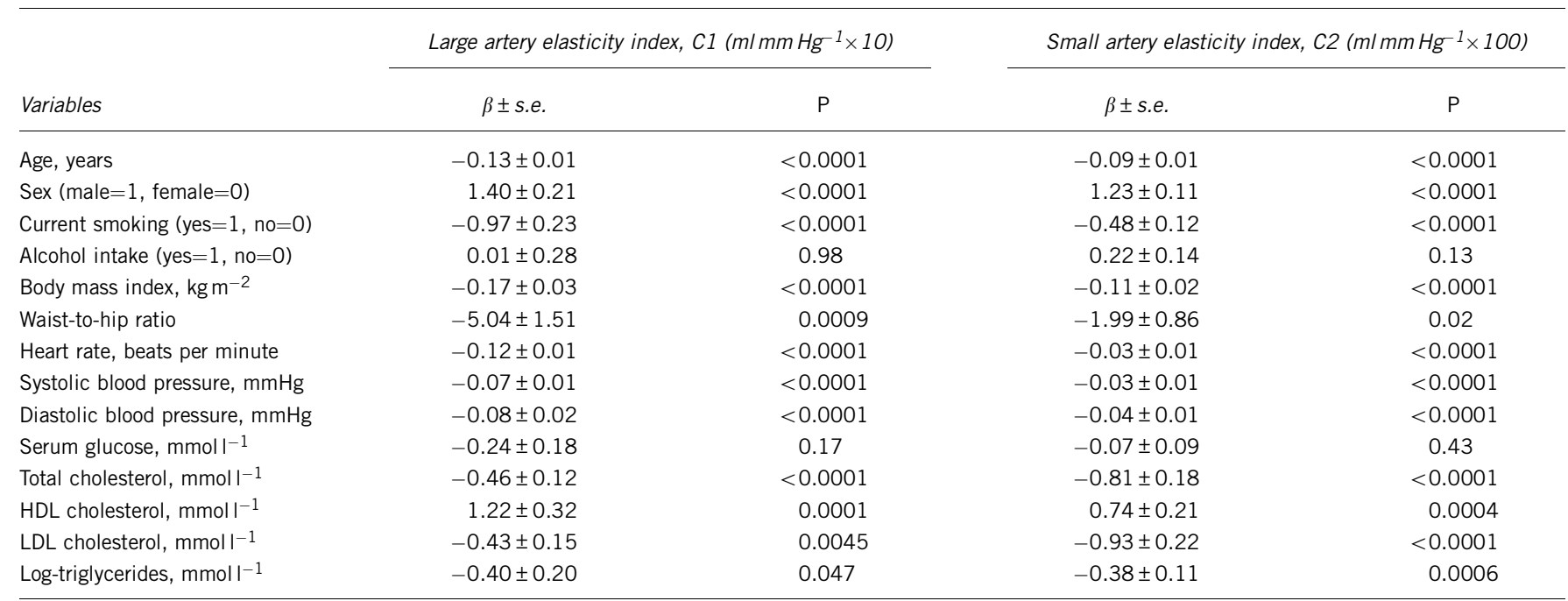

Abbreviations: HDL, high-density lipoprotein; LDL, low-density lipoprotein; log-triglycerides, log-transformed of triglycerides.
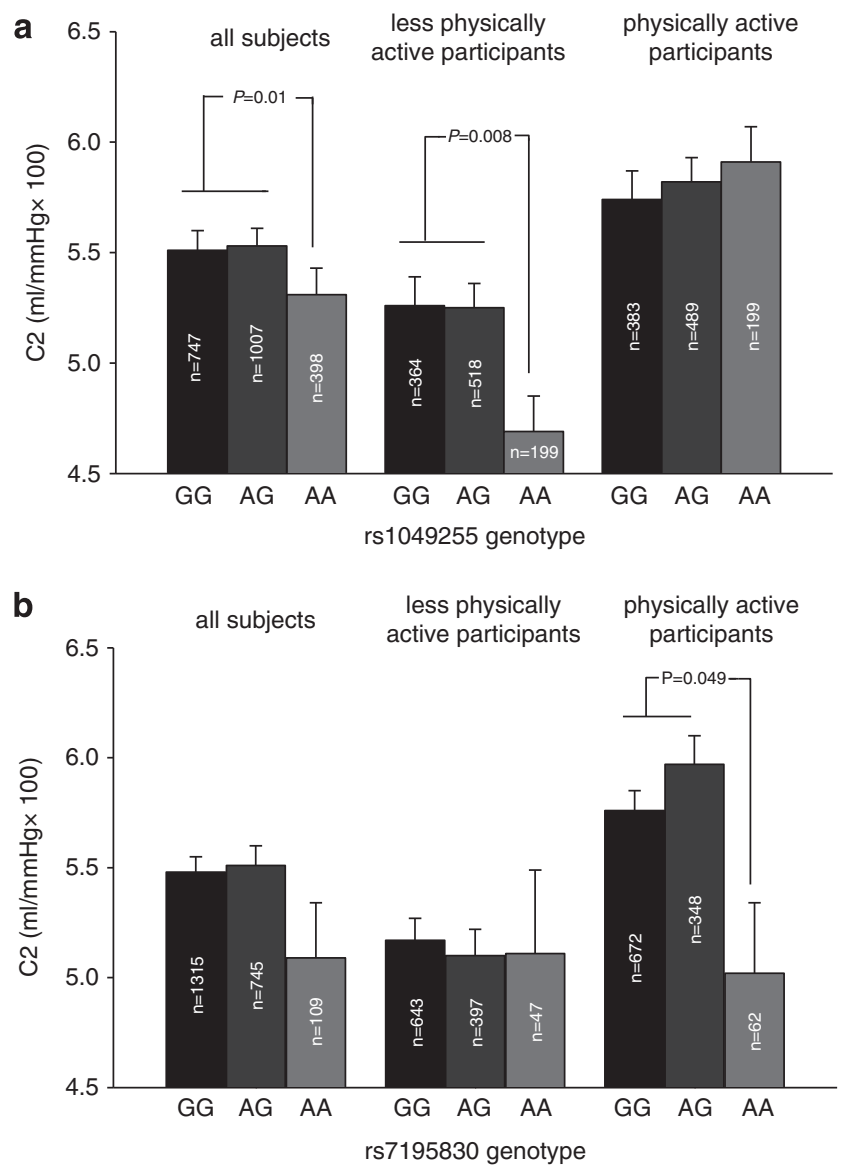

Figure 1 Small artery elasticity (C2) of the study groups stratified by the genotypes of the rs1049255 polymorphism (a) and the rs7195830 polymorphism (b) in the study population (left-hand panel), in less physically active participants (middle panel) and in physically active participants (right-hand panel). The values are the means \pm s.e. adjusted for age, sex, heart rate, smoking status, waist-to-hip ratio, body mass index, systolic blood pressure, diastolic blood pressure, total cholesterol level, HDL cholesterol level, LDL cholesterol level and triglyceride levels. $P$-values were calculated by multiple regression analysis. to have higher C2 values. Notably, we also found an interaction between the rs7195830 polymorphism and energy expenditure. The GG/AG genotype of the rs7 195830 polymorphism was associated with higher $\mathrm{C} 2$ values in physically active participants.

Accumulating evidence indicates that the increased superoxide production accounts for a significant proportion of endothelial dysfunction. ${ }^{27,28}$ Schirmer et al. ${ }^{29}$ suggested that superoxide production is associated with rs1049255 genotypes, being higher in AA carriers compared with GG carriers. Accordingly, we found similar results for the $\mathrm{C} 2$ response across the genotypes, with a significant difference observed between AA and GG/AG carriers. But MaciasReyes et al. ${ }^{10}$ found opposite results, whereas Wyche et al. ${ }^{12}$ failed to find any relationship. Accordingly, associations between the rs1049255 polymorphism and vascular complications have been contradictory. ${ }^{9,10}$ These conflicting results might be due to differences in the sample size, the ethnicity of the population studied and/or the oxidative stress status of the individuals. Regular physical activity has been reported to be the most effective non-pharmacological intervention to enhance endogenous antioxidant capacity and alleviate oxidative stress-induced tissue damage. ${ }^{30}$ Our findings on the interaction between variants of the CYBA gene and physical activity were in line with the results of a recently published study ${ }^{15}$ in which 59 apparently healthy Caucasian men and women participated in an exercise training program. Carriers of the A allele of the rs1049255 polymorphism displayed a significantly greater decrease in superoxide production after exercise training compared with the A allele noncarrier group. A significant interaction effect between exercise training and the rs1049255 polymorphism with respect to changes in superoxide production was also found. The results of the previous study and the present study suggested that in research on oxidative stressrelated phenotypes, energy expenditure should be taken into account.

Recently, haplotype association analysis has been suggested as a more powerful approach for identifying genes that predispose individuals to complex conditions, compared with the analysis of any individual SNP. ${ }^{31}$ The two SNPs in the present study were located in the P22phox subunit of the CYBA gene and were in linkage disequilibrium as described in the HAPMAP database. Although the rs7195830 polymorphism was not related to arterial elasticity in all subjects, the rs1049255G-rs7195830G haplotype was found to be 
Table 3 Multiple regression analysis of haplotypes on arterial elasticity indices

\begin{tabular}{|c|c|c|c|c|c|}
\hline \multirow[b]{2}{*}{ Haplotypes } & \multirow[b]{2}{*}{ Frequency } & \multicolumn{2}{|c|}{$\begin{array}{l}\text { Large artery elasticity index, } \\
\qquad \mathrm{Cl}\left(\mathrm{m} / \mathrm{mm} \mathrm{Hg}^{-1} \times 10\right)\end{array}$} & \multicolumn{2}{|c|}{$\begin{array}{l}\text { Small artery elasticity index, } \\
\qquad 2\left(\mathrm{~m} / \mathrm{mm} \mathrm{Hg}^{-1} \times 100\right)\end{array}$} \\
\hline & & $\beta \pm s . e^{a}$ & $\mathrm{P}$ & $\beta \pm s . e^{a}$ & $P$ \\
\hline rs1049255A-rs7195830G & 0.41 & $-0.12 \pm 0.18$ & 0.52 & $-0.01 \pm 0.09$ & 0.90 \\
\hline rs1049255G-rs7195830G & 0.37 & $0.28 \pm 0.21$ & 0.18 & $0.33 \pm 0.10$ & 0.0015 \\
\hline rs1049255G-rs7195830A & 0.21 & $0.11 \pm 0.18$ & 0.54 & $-0.03 \pm 0.09$ & 0.76 \\
\hline
\end{tabular}

${ }^{a}$ Adjusted for age, sex, heart rate, smoking status, waist-to-hip ratio, body mass index, systolic blood pressure, diastolic blood pressure, total cholesterol level, high-density lipoprotein cholesterol level, low-density lipoprotein cholesterol level and triglyceride levels.

associated with C2. Remarkably, the regression coefficient from the haplotype analysis was greater than that of either SNP, so it was obvious that such an association was the result of the combined action of both loci.

It is notable that the present study found a relationship between the rs7195830 polymorphism and arterial elasticity in physically active participants. The few studies on the rs7195830 polymorphism have not elucidated the mechanisms underlying this result, but this polymorphism was also selected as an oxidative stress-related locus in several previous studies on non-Hodgkin lymphoma and cervical cancer. ${ }^{13,14}$ It is possible that the rs7195830 polymorphism, which is located in the $3^{\prime}$-untranslated region, might influence messenger RNA stability or modulate the transcription rate and affect reactive oxygen species generation. Further investigations on the function of this polymorphism are necessary.

In addition, we only found a genetic association with $\mathrm{C} 2$, but not with $\mathrm{C} 1$, in the apparently healthy participants, suggesting that $\mathrm{C} 2$ might be an earlier indicator of endothelial dysfunction than $\mathrm{C} 1$, which was in accordance with previous studies. ${ }^{4,32}$

Our present study should be interpreted within the context of its limitations. First, our population-based study suggested a genetic association with arterial elasticity indices that could be modified by physical activity but did not directly measure the biochemical indices related to superoxide production to verify our hypothesis. Second, we did not perform an intervention study of physical activity that could reflect the effect of energy expenditure on polymorphisms directly; however, our results were in accordance with the previous study, ${ }^{15}$ which used the interventional method to evaluate the physical exercise. Furthermore, our definition of physical activity was based on the data of the present study; nevertheless, the cutoff point for the division of physically active and less active participants was similar to the mean energy expenditure in Chinese residents with occupations requiring a moderate amount of physical activity $(2581 \mathrm{kcal}$ per day). ${ }^{33}$ Finally, our study was cross-sectional, and hence no causal inference could be drawn.

In conclusion, the results of our study suggest a functional role of the rs1049255 and rs7195830 polymorphisms of the CYBA gene in the modulation of arterial elasticity measured by the $\mathrm{C} 1$ and $\mathrm{C} 2$ indices. This relationship could be modified by physical activity in an apparently healthy Chinese population. Our findings require confirmation by prospective and interventional studies, and the mechanisms underlying the association between the rs7195830 polymorphism and arterial elasticity in physically active participants remain to be elucidated.

\section{CONFLICT OF INTEREST}

The authors declare no conflict of interest.

\section{ACKNOWLEDGEMENTS}

We wish to thank Prof. Jay N Cohn for valuable revision. The study was supported by grants from the Ministry of Science and Technology, P.R.C (2007BAI07A10, 2008BAI65B09) and Project Supported by the Department of Science and Technology of Jiangsu Province, China (BE2009613).

All the investigators agreed the order in which the contributors are listed, and the first two listed made the greatest contribution to the paper.

1 Bonetti PO, Lerman LO, Lerman A. Endothelial dysfunction: a marker of atherosclerotic risk. Arterioscler Thromb Vasc Biol 2003; 23: 168-175.

2 Tao J, Jin YF, Yang Z, Wang LC, Gao XR, Lui L, Ma H. Reduced arterial elasticity is associated with endothelial dysfunction in persons of advancing age: comparative study of noninvasive pulse wave analysis and laser Doppler blood flow measurement. Am J Hypertens 2004; 17: 654-659.

3 Gilani M, Kaiser DR, Bratteli CW, Alinder C, Rajala S, Bank AJ, Cohn JN. Role of nitric oxide deficiency and its detection as a risk factor in pre-hypertension. J Am Soc Hypertens 2007; 1: 45-55.

4 Duprez DA, Jacobs Jr DR, Lutsey PL, Bluemke DA, Brumback LC, Polak JF, Peralta CA, Greenland P, Kronmal RA. Association of small artery elasticity with incident cardiovascular disease in older adults: the multi-ethnic study of atherosclerosis. Am J Epidemiol 2011; 174: 528-536.

5 Kampus P, Kals J, Unt E, Zilmer K, Eha J, Teesalu R, Normak A, Zilmer M. Association between arterial elasticity, C-reactive protein and maximal oxygen consumption in welltrained cadets during three days extreme physical load: a pilot study. Physiol Meas 2008; 29: 429-437.

6 Zhou L, Chen Y, Sun N, Liu X. Family history of hypertension and arterial elasticity characteristics in healthy young people. Hypertens Res 2008; 31: 833-839.

7 Mueller CF, Laude K, McNally JS, Harrison DG. Redox mechanisms in blood vessels. Arterioscler Thromb Vasc Biol 2005; 25: 274-278.

8 Ushio-Fukai M, Zafari AM, Fukui T, Ishizaka N, Griendling KK. p22phox is a critical component of the superoxide-generating NADH/NADPH oxidase system and regulates angiotensin II-induced hypertrophy in vascular smooth muscle cells. J Biol Chem 1996; 271: 23317-23321.

9 Zafari AM, Davidoff MN, Austin H, Valppu L, Cotsonis G, Lassegue B, Griendling KK. The A640G and C242T p22(phox) polymorphisms in patients with coronary artery disease. Antioxid Redox Signal 2002; 4: 675-680.

10 Macias-Reyes A, Rodriguez-Esparragon F, Caballero-Hidalgo A, Hernandez-Trujillo $Y$, Medina A, Rodriguez-Perez JC. Insight into the role of CYBA A640G and C242T gene variants and coronary heart disease risk. A case-control study. Free Radic Res 2008; 42: 82-92.

11 Gardemann A, Mages P, Katz N, Tillmanns H, Haberbosch W. The p22 phox A640G gene polymorphism but not the $\mathrm{C} 242 \mathrm{~T}$ gene variation is associated with coronary heart disease in younger individuals. Atherosclerosis 1999; 145: 315-323.

12 Wyche KE, Wang SS, Griendling KK, Dikalov SI, Austin H, Rao S, Fink B, Harrison DG, Zafari AM. C242T CYBA polymorphism of the NADPH oxidase is associated with reduced respiratory burst in human neutrophils. Hypertension 2004; 43: 1246-1251.

13 Lan Q, Zheng T, Shen M, Zhang Y, Wang SS, Zahm SH, Holford TR, Leaderer B, Boyle P, Chanock S. Genetic polymorphisms in the oxidative stress pathway and susceptibility to non-Hodgkin lymphoma. Hum Genet 2007; 121: 161-168.

14 Wang SS, Bratti MC, Rodriguez AC, Herrero R, Burk RD, Porras C, Gonzalez P, Sherman ME, Wacholder S, Lan ZE, Schiffman M, Chanock SJ, Hildesheim A. Common variants in immune and DNA repair genes and risk for human papillomavirus persistence and progression to cervical cancer. J Infect Dis 2009; 199: 20-30.

15 Park JY, Ferrell RE, Park JJ, Hagberg JM, Phares DA, Jones JM, Brown MD. NADPH oxidase p22phox gene variants are associated with systemic oxidative stress biomarker responses to exercise training. J Appl Physiol 2005; 99: 1905-1911.

16 Craig CL, Marshall AL, Sjöström M, Bauman AE, Booth ML, Ainsworth BE, Pratt M, Ekelund U, Yngve A, Sallis JF, Oja P. International physical activity questionnaire: 12-country reliability and validity. Med Sci Sports Exerc 2003; 35: 1381-1395. 
17 Deng HB, Macfarlane DJ, Thomas GN, Lao XQ, Jiang CQ, Cheng KK, Lam TH. Reliability and validity of the IPAQ-Chinese: the Guangzhou Biobank Cohort study. Med Sci Sports Exerc 2008; 40: 303-307.

18 McArdle WD, Katch FI, Katch VL (eds). Exercise Physiology: Energy, Nutrition and Human Performance, 3rd edn. Lea \& Febiger: Philadelphia, Pennsylvania, 1991 , pp 136.

19 Ainsworth BE, Haskell WL, Whitt MC, Irwin ML, Swartz SJ, O'Brien WL, Bassett Jr DR, Schmitz KH, Emplaincourt PO, Jacobs Jr DR, Leon AS. Compendium of physical activities: an update of activity codes and MET intensities. Med Sci Sports Exerc 2000; 32: S498-S504.

$20 \mathrm{Li} \mathrm{LH}, \mathrm{Li}$ Y, Wen Y, Wang JG. Anthropometric and metabolic phenotypes in relation to the ADRA2B deletion/insertion polymorphism in Chinese population. $J$ Hypertens 2008; 26: 2161-2167.

21 Glasser SP, Arnett DK, McVeigh GE, Finkelstein SM, Bank AJ, Morgan DJ, Cohn JN. Vascular compliance and cardiovascular disease: a risk factor or a marker? Am J Hypertens 1997; 10: 1175-1189.

22 Cohn JN. Pathophysiologic and prognostic implications of measuring arterial compliance in hypertensive disease. Prog Cardiovasc Dis 1999; 41: 441-450.

23 Zimlichman R, Shargorodsky M, Boaz M, Duprez D, Rahn KH, Rizzoni D, Payeras AC, Hamm C, McVeigh G. Determination of arterial compliance using blood pressure waveform analysis with the CR-2000 system: reliability, repeatability, and establishment of normal values for healthy European population-the seven European sites study (SESS). Am J Hypertens 2005; 18: 65-71.

24 Ge JY, Li XL, Zhang HF, Xu Q, Tong M, Wang JG. Elasticity indices of large and small arteries in relation to the metabolic syndrome in Chinese. Am J Hypertens 2008; 21 : 143-147.
25 Brumback LC, Jacobs Jr DR, Dermond N, Chen H, Duprez DA. Reproducibility of arterial elasticity parameters derived from radial artery diastolic pulse contour analysis: the multi-ethnic study of atherosclerosis. Blood Press Monit 2010; 15: 312-315.

26 Livak KJ. Allelic discrimination using fluorogenic probes and the $5^{\prime}$ nuclease assay. Genet Anal 1999; 14: 143-149.

27 Wind S, Beuerlein K, Armitage ME, Taye A, Kumar AH, Janowitz D, Neff C, Shah AM, Wingler K, Schmidt HH. Oxidative stress and endothelial dysfunction in aortas of aged spontaneously hypertensive rats by NOX1/2 is reversed by NADPH oxidase inhibition. Hypertension 2010; 56: 490-497.

28 Touyz RM, Briones AM. Reactive oxygen species and vascular biology: implications in human hypertension. Hypertens Res 2011; 34: 5-14.

29 Schirmer M, Hoffmann M, Kaya E, Tzvetkov M, Brockmoller J. Genetic polymorphisms of $\mathrm{NAD}(\mathrm{P}) \mathrm{H}$ oxidase: variation in subunit expression and enzyme activity. Pharmacogenomics J 2008; 8: 297-304.

30 Miyazaki H, Oh-ishi S, Ookawara T, Kizaki T, Toshinai K, Ha S, Haga S, Ji LL, Ohno H. Strenuous endurance training in humans reduces oxidative stress following exhausting exercise. Eur J Appl Physiol 2001; 84: 1-6.

31 Ginsburg GS, Shah SH, McCarthy JJ. Taking cardiovascular genetic association studies to the next level. J Am Coll Cardiol 2007; 50: 930-932.

32 Grey E, Bratteli C, Glasser SP, Alinder C, Finkelstein SM, Lindgren BR, Cohn JN. Reduced small artery but not large artery elasticity is an independent risk marker for cardiovascular events. Am J Hypertens 2003; 16: 265-269.

33 Yao M, McCrory MA, Ma G, Li Y, Dolnikowski GG, Roberts SB. Energy requirements of urban Chinese adults with manual or sedentary occupations, determined using the doubly labeled water method. Eur J Clin Nutr 2002; 56: 575-584. 